\title{
Mesure de débit en rivière par station radar hauteur / vitesse selon la méthode de la vitesse témoin
}

\author{
Fabien THOLLET ${ }^{1}$, Jérôme LE COZ1, Guillaume DRAMAIS ${ }^{1}$, Guillaume NORD², Raphaël LE BOURSICAUD², \\ Elise $\mathrm{JACOB}^{4}$ Alexis BUFFET ${ }^{1}$ \\ ${ }^{1}$ IrsteaUR HHLY, 5 rue de la Doua 69100 Villeurbanne, France-fabien.thollet@irstea.fr \\ ${ }^{2}$ Laboratoire d'Etude des Transferts en Hydrologie et Environnement, Université Grenoble-Alpes/CNRS, IRD, Grenoble, France - \\ guillaume.nord@ujf-Grenoble.fr \\ ${ }^{3}$ CNR 4 rue de Chalon sur Saône-69007Lyon, France - r.leboursicaud@cnr.tm.fr@cnr.tm.fr \\ ${ }^{4}$ DRIEE Ile-de-France - SPRN/PHPC/Unité Hydrologie Réseau Mesure 10 rue Crillon 75194 PARIS Cedex $04-$ \\ elise.jacob@developpement-durable.gouv.fr
}

\begin{abstract}
RÉSUMÉ. - Depuis quelques années, des stations radar hauteur-vitesse ont été mises en service en France. Ce type de station fournit une mesure de hauteur d'eau et de vitesse de surface en un point fixe de la section. Le débit est calculé comme le produit de la surface mouillée et de la vitesse débitante. La surface mouillée est obtenue via une relation hauteur-surface $S(h)$ établie à partir d'un profil bathymétrique ; et la vitesse débitante est calculée via une relation entre la vitesse mesurée par radar $\left(V_{i}\right)$ et la vitesse moyenne dans la section $\left(V_{m o y}\right)$, établie à partir de jaugeages. Il s'agit de la «méthode de la vitesse témoin» ou « index velocity method».

Comme nous le verrons au travers d'exemples sur différentes stations, la méthode présente des avantages par rapport aux courbes de tarage hauteur-débit :

- les extrapolations en crue sont mieux documentées, la vitesse évoluant plus faiblement que le débit avec la hauteur et la relation hauteur-surface étant connue par ailleurs.

- la gestion des détarages est plus facile car la relation $V_{\text {moy }}\left(V_{i}\right)$ évolue peu et la relation $S(h)$ est facile à recaler avec un seul levé bathymétrique.

- l'évolution de la relation $V_{i}(h)$, mesurée en temps réel, permet de détecter les anomalies, signes de détarages potentiels de la station.

L'utilisation plus large de la méthode et le développement des instruments de mesure permettra à l'avenir de mieux évaluer l'incertitude liée au calcul du débit. Des perspectives pour l'analyse bayésienne des stations vitesse témoin sont discutées.
\end{abstract}

Mots-clés : vitesse témoin, radar, débit, courbe de tarage, hydrométrie

\section{Streamflow monitoring at stage / velocity radar stations using the index velocity method}

\begin{abstract}
In recent years, stage / velocity radar stations have been installed in France. This type of stations provides measurements of water level and surface velocity at a fixed point of the cross-section. Discharge is calculated as the product of the wetted area and cross-sectional average velocity; respectively obtained using a stage-area scale $S(h)$ established from a bathymetry profile, and a relation between the index velocity measured by the radar $\left(V_{i}\right)$ and the cross-sectional average velocity $\left(V_{\text {mean }}\right)$, established with gaugings. This is the «index velocity method ».

As discussed through various examples, the index velocity method has advantages over traditional stage-discharge rating curves:

- Flood extrapolations are better known: velocity varies more slowly than water discharge with stage and the stage-area relation can be predicted.

- Managing bed evolution is easier because the $V_{\text {mean }}\left(V_{i}\right)$ relation changes little and the $S(h)$ relation is easy to reset with a bathymetry survey.

- Monitoring the $V_{i}(h)$ measured relation in real time allows detecting rating changes of the station.

The wider use of the method and the development of measurement instruments will allow the future assessment of the uncertainty related to the calculation of water discharge. Perspectives for the bayesian analysis of index velocity ratings are discussed.
\end{abstract}

Key-words: index velocity method, radar, water discharge, rating curve, hydrometry

\section{INTRODUCTION}

La méthode de la vitesse témoin ${ }^{1}$ ( en anglais) a été développée par 1'USGS (United States Geological Survey), et

1. Nous adoptons cette terminologie française car elle est fidèle à l'original anglais et communément utilisée au Canada francophone. est utilisée sur plus de 470 stations américaines [Levesque et al., 2012]. Son utilisation est particulièrement appropriée pour des sites où la relation hauteur-débit n'est pas univoque, à cause d'une influence aval variable (barrages, marée, confluences notamment), ou éventuellement à cause d'un effet d'hystérésis en régime transitoire. La vitesse est le plus souvent mesurée par des systèmes acoustiques immergés : 
profileurs Doppler ADCP fixes horizontaux ou verticaux [Le Coz et al., 2009], cordes à ultrasons à temps de transit, débitmètres Doppler à émission continue [Birgand et al., 2005]. Mais la méthode s'applique également bien aux systèmes de mesure sans contact hauteur-vitesse par radars aériens.

En effet, les techniques de vélocimétrie sans contact par radar se démocratisent et les équipes d'hydrométrie les utilisent de plus en plus pour les jaugeages [Nord et al., 2016], [Welber et al., 2016], mais aussi pour équiper des stations [Costa et al., 2006]. En France, les services opérationnels exploitent plusieurs stations de ce type, majoritairement sur des torrents ou rivières de montagne; la méthode étant particulièrement adaptée à ce type de cours d'eau souvent difficiles à jauger par les méthodes conventionnelles, notamment en présence de transport sédimentaire [Nord et al., 2014]. Toutefois, des grands cours d'eau comme le Rhône à Lyon ou le Rhin à Hoechst ont également été équipés de ce type de station car elles permettent de réduire la maintenance et les coûts associés en augmentant la durabilité du matériel et offrent davantage de sécurité pour les opérateurs et le matériel moins vulnérables notamment en crue.

Nous présentons ici les outils et conditions favorables à la réalisation de bonnes mesures puis les différentes étapes pour le calcul du débit par la méthode de la vitesse témoin. Enfin au travers de résultats expérimentaux nous illustrerons ses avantages par rapport aux méthodes traditionnelles de mesure de débit par relation hauteur/débit, principalement sur la mise en évidence de détarages et la construction d'une nouvelle relation.

\section{LES MESURES ÉLÉMENTAIRES DE HAUTEUR ET DE VITESSE PAR RADAR}

\section{II.1. Principes de mesure}

Les radars sans contact hauteur / vitesse combinent deux technologies. Le niveau d'eau est mesuré à l'aide d'un capteur fixe pointant verticalement vers l'écoulement. Le temps de transit aller-retour de l'onde radar émise, ou temps de vol, est proportionnel à la distance entre l'émetteur et la surface réfléchissante. La détermination de la vitesse d'écoulement est basée sur le principe de décalage de fréquence (effet Doppler). Le radar de vitesse, incliné selon un angle de $30^{\circ}$ à $60^{\circ}$ sous l'horizontale [Dramais et al., 2011]; [Welber et al., 2016], génère un signal électromagnétique qui est rétrodiffusé par les rugosités de la surface de l'écoulement vers le récepteur avec un décalage de fréquence proportionnel à la vitesse (figure 1a).

\section{II.2. Déploiement de l’instrumentation}

\section{I.1.1. Site de mesure et instrumentation}

Le premier critère à considérer pour le déploiement est l'accès à la station de mesure en toutes circonstances notamment en crue. L'accès à la rivière et aux instruments doit être sécurisé pour faciliter les interventions de maintenance, de jaugeages et de mesures bathymétriques. Les radars étant placés en hauteur, il est nécessaire d'utiliser des dispositifs antichute (barrières, harnais, nacelles négatives...) lors des opérations de maintenance et que celles-ci soient effectuées par du personnel habilité et formé.

Du point de vue hydraulique, il faut trouver une section rectiligne garantissant un écoulement le plus uniforme possible avec une section de contrôle stable. Ces conditions doivent si possible être vérifiées sur le terrain à différents régimes. La mesure de hauteur par le radar doit pouvoir être vérifiée et calée contre l'échelle limnimétrique de référence de la station.

La proximité d'un pont facilitera la fixation des radars mesurant hauteur d'eau et vitesse de surface, en s'assurant toutefois que le trafic routier n'induise pas trop de vibrations sur le matériel ce qui pourrait influencer la mesure. Il est donc prudent d'exclure les ponts ferroviaires et d'être vigilant avec les ponts suspendus ou sujets à déformation par dilatation thermique. Une pile de pont influencera le champ de vitesse de surface [Dramais et al., 2011]; il convient donc de s'assurer que la mesure n'est pas dans la zone d'influence à tous régimes. En l'absence de pont, on peut envisager la solution de bras déportés depuis la berge et montés sur pivot pour faciliter la récupération du matériel (figure 1b). Ils offrent de bonnes conditions de stabilité des instruments (position et angle de visée). Néanmoins, les longueurs de déport sont limitées pour éviter des bras de levier trop importants (2 à 3 mètres maximum) et la mesure de vitesse doit rester représentative de l'écoulement moyen sur la section, donc dans la veine principale d'écoulement idéalement. Ces systèmes s'appliqueront plus facilement sur des cours d'eau peu larges pour lesquels l'écoulement est toujours suffisant pour que les radars ne visent pas une zone sèche ou des obstacles.

Enfin selon l'angle d'inclinaison de l'appareil (de 30 à $60^{\circ}$ ) et l'angle d'ouverture du faisceau émis par le radar (de l'ordre de $10^{\circ}$, selon les modèles), la mesure est moyennée sur une surface (ellipse) qui varie en fonction du niveau d'eau. Augmenter l'angle d'inclinaison améliore l'intensité rétrodiffusée, donc la qualité du signal reçu, mais augmente aussi les erreurs de projection des vitesses. Plus un radar est fixé haut, plus l'empreinte du faisceau de mesure est importante. Et il en va de même pour de faibles angles d'inclinaison. Le tableau 1 donne des indications sur la surface mesurée pour le cas d'un radar Sommer RQ24.

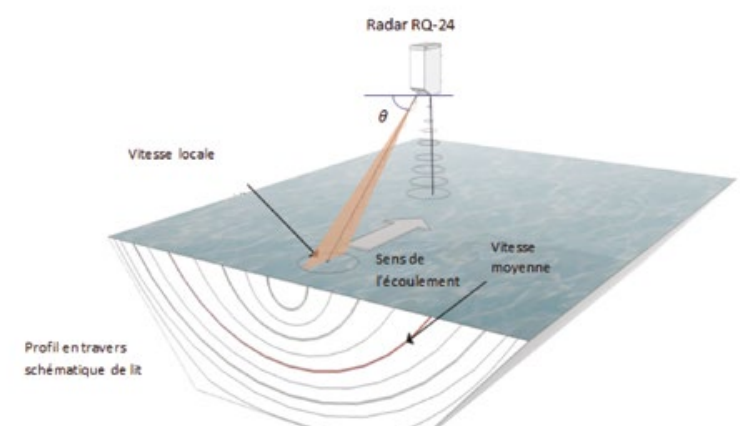

Figure 1a : principe de mesure d'une station radar hauteur vitesse (extrait documentation Sommer RQ24)

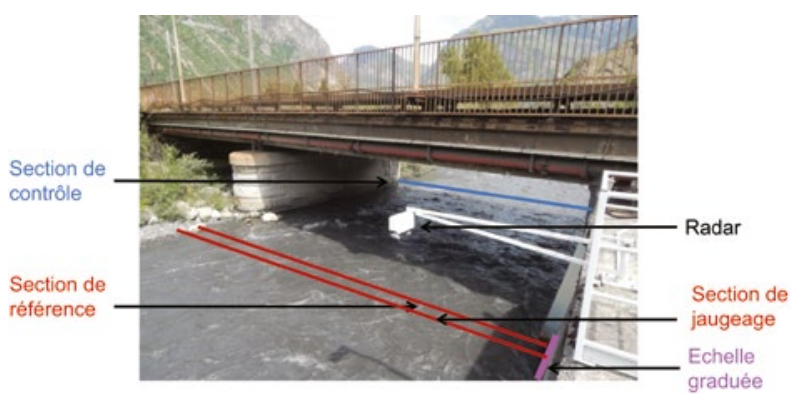

Figure1b : station hydrométrique sur l'Arvan (Photo: Jacob 2014) 
Tableau 1 : Caractéristiques de l'empreinte du faisceau pour un radar Sommer (angle d'émission 10)

\begin{tabular}{cccc}
\hline $\begin{array}{c}\text { Hauteur radar - } \\
\text { surface eau } \\
\text { en } \mathrm{m}\end{array}$ & $\begin{array}{c}\text { Angle d'inclinaison } \\
\text { en }\end{array}$ & $\begin{array}{c}\text { Diamètre du disque } \\
\text { en } \mathrm{m}\end{array}$ & $\begin{array}{c}\text { Surface du disque } \\
\text { en }^{2}\end{array}$ \\
\hline 3 & 40 & 0,57 & 0,26 \\
\hline 3 & 50 & 0,42 & 0,14 \\
\hline 3 & 60 & 0,33 & 0,09 \\
\hline 10 & 40 & 1,92 & 2,89 \\
\hline 10 & 50 & 1,39 & 1,52 \\
\hline 10 & 60 & 1,11 & 0,97 \\
\hline
\end{tabular}

\section{I.1.2. Influence $d u$ vent}

La mesure radar nécessite une rugosité de surface de l'écoulement pour qu'il renvoie un écho suffisant dans la direction du radar, c'est-à-dire des vaguelettes ou bouillons. Les conditions d'écoulement doivent donc être dynamiques pour éviter « l'effet miroir » du cours d'eau. L'influence du vent sur la surface libre est à considérer [Costa et al., 2006]. Elle dépend de son amplitude et de sa direction, et peut devenir négligeable pour des écoulements avec de fortes vitesses d'écoulement, typiquement supérieures à $1 \mathrm{~m} / \mathrm{s}$.

La station de Perrache sur le Rhône à Lyon est équipée d'un radar Sommer RQ30. Les données peuvent être comparées à celles issues d'une station de débit $Q(h)$ toute proche et d'une station météo enregistrant notamment orientation et vitesse du vent (figure 2). Cette dernière est projetée selon l'axe Nord/Sud qui correspond à l'orientation du fleuve à Lyon. Les valeurs de débit issues des deux stations corrèlent particulièrement bien lorsque la vitesse du vent est nulle. Pour des vitesses non nulles, on voit apparaitre « un effet vent » comme par exemple le 7 février 2016 où un vent Sud/ Nord de 7 à $8 \mathrm{~km} / \mathrm{h}$ induit une erreur d'environ $30 \%$ sur la mesure du débit (sous-estimation). Globalement, cet effet est significatif sur ce cours d'eau (fluvial) dès $5 \mathrm{~km} / \mathrm{h}$, y compris pour des débits importants de l'ordre de $1000 \mathrm{~m}^{3} / \mathrm{s}$. Il serait intéressant de vérifier si l'impact est toujours manifeste sur un cours d'eau torrentiel.

\section{LA MÉTHODE DE LA VITESSE TÉMOIN}

\section{III.1. Principe général}

La vitesse de surface de l'écoulement $V_{i}$ est mesurée en un point du cours d'eau. Cette vitesse est reliée à la vitesse débitante $V_{\text {mov }}$ sur la section de mesure. On mesure en parallèle la hauteur d'eau h pour déterminer $S$, la surface mouillée sur la section. Le produit de ces deux valeurs permet d'obtenir le débit $Q$ (Eq.1) :

$$
Q=V_{\text {moy }}\left(V_{i}\right) \times S(h)
$$

où $Q$ indique le débit, $V_{m o y}$ la vitesse moyenne de l'écoulement dans la section du radar, $V_{i}$ la vitesse issue du radar et $S(h)$ la relation qui donne la surface mouillée en fonction de la hauteur d'eau dans la section du radar.

Le calage de la relation $V_{\text {moy }}\left(V_{i}\right)$ s'appuie sur des jaugeages réalisés dans la section du radar ; la vitesse moyenne de l'écoulement est donc bien celle du jaugeage. Comme il n'est pas toujours possible de jauger dans la section de mesure de la station hydrométrique (typiquement pour des problèmes d'accessibilité, ou lorsque les mesures sont réalisées au camion jaugeur depuis un pont faiblement éloigné...) le débit instantané déterminé par le jaugeage ne donne pas directement la vitesse moyenne de l'écoulement

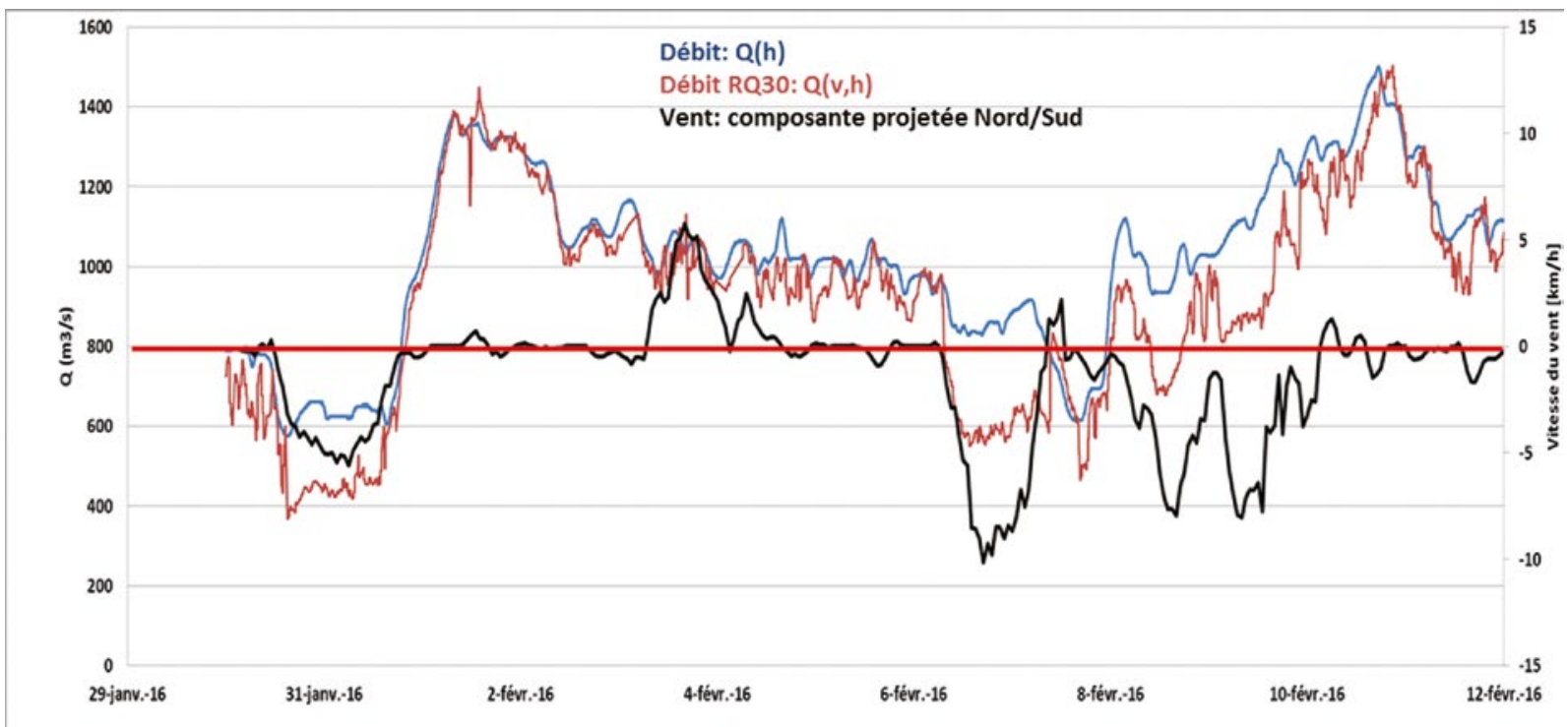

Figure 2 : Débit $Q(h)$ et débit $Q(v, h)$ en lien avec la vitesse du vent sur le Rhône à Lyon 
dans la section du radar mais permet de la recalculer en utilisant la section mouillée de l'échelle limnimétrique (Eq.2) :

$$
V_{\text {moy }}=Q_{\text {jaugeage }} / S(h)
$$

Il est possible d'avoir recours à la modélisation numérique pour le calage de la relation $V_{m o v}\left(V_{i}\right)$ mais des jaugeages restent dans tous les cas utiles et nécessaires pour vérifier la relation.

Tout changement dans la répartition spatiale des vitesses au sein de la section, en particulier en cas de débordement dans le lit majeur, changement de contrôle hydraulique ou encore au cours des cycles de marée, affecte la relation $V_{m o v}\left(V_{i}\right)$. Aussi l'extrapolation de la relation doit être faite avec prudence. Il faut alors vérifier la stabilité de la relation selon la gamme de débits et l'application de plusieurs relations est possible.

On peut aussi mesurer en continu plusieurs vitesses de surface dans la section. Elles sont reliées à la vitesse débitante $V_{\text {moy }}$ selon le même principe que décrit ci-dessus où le cours d'eau peut être divisé en sous-sections, par exemple pour distinguer un écoulement rapide dans le lit mineur d'un écoulement plus lent dans le lit majeur en crue. Le calcul du débit global est la somme des débits partiels dans chaque sous-section. Pour un grand nombre de points, on arrive progressivement à une méthode d'exploration partielle du champ des vitesses (méthode employée pour les stations LSPIV) [Hauet et al., 2009].

Pour calculer le débit en continu par la méthode de la vitesse témoin, il convient de calculer une relation hauteur / surface mouillée (Eq.1). Un levé bathymétrique détaillé doit donc être réalisé sur une section de référence perpendiculaire à l'écoulement (figure $1 \mathrm{~b}$ ) et située aussi proche que possible du radar hauteur / vitesse [Levesque et Oberg, 2012]. Une surveillance régulière de la bathymétrie de la section doit être mise en place afin de détecter un éventuel détarage qui aurait pour conséquence le changement de la table hauteur / surface mouillée. Des changements répétés de la forme de la section de référence sont le signe de l'inadéquation de cette section avec la méthode de la vitesse témoin.

\section{III.2. Détermination de la surface mouillée}

L'établissement du barème hauteur / surface mouillée peut être effectué grâce à des logiciels d'hydraulique. Levesque et Oberg, [2012] utilisent le logiciel AreaComp développé à l'USGS (https://hydroacoustics.usgs.gov/indexvelocity/ AreaComp.shtml); d'autres logiciels d'hydraulique comme PamHyr développé à Irstea permettent également l'établissement de ce barème (figure $3 \mathrm{a}$ ).

Nous proposons une approche pour calculer une relation $S(h)$ et estimer un fuseau d'incertitudes en utilisant la méthode BaRatin et son interface BaRatinage [Le Coz et al., 2014]. Cette méthode a été développée pour établir des relations hauteur / débit et en estimer l'incertitude de façon rigoureuse en s'appuyant sur l'inférence bayésienne. Elle est ici détournée de son application principale mais s'applique parfaitement à ce type de courbes d'étalonnage. Sur l'Arvan à Saint Jean de Maurienne, nous avons utilisé des couples Hauteur / Surface mouillée issus de 4 levés bathymétriques, afin de prendre en compte les erreurs liées aux évolutions de la section de référence, en plus des erreurs de mesure (figure $3 b$ ). On cale une loi puissance $S(h)$, Eq. 3, par morceaux pour chaque tronçon de lit représenté par un chenal rectangulaire équivalent.

$$
S(h)=a(h-b)^{c}
$$

où $a$ indique la largeur du rectangle équivalent, $b$ la cote du fond et le coefficient $c$ forcé à 1 pour un chenal rectangulaire.

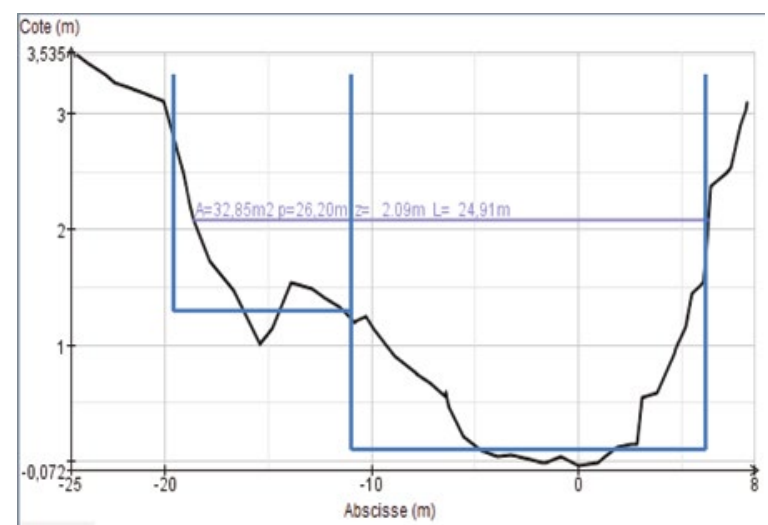

Figure 3a : profil Abscisse / Cote sur la station de la Claduègne avec le logiciel Pamhyr et les rectangles équivalents pour la relation $S(h)$

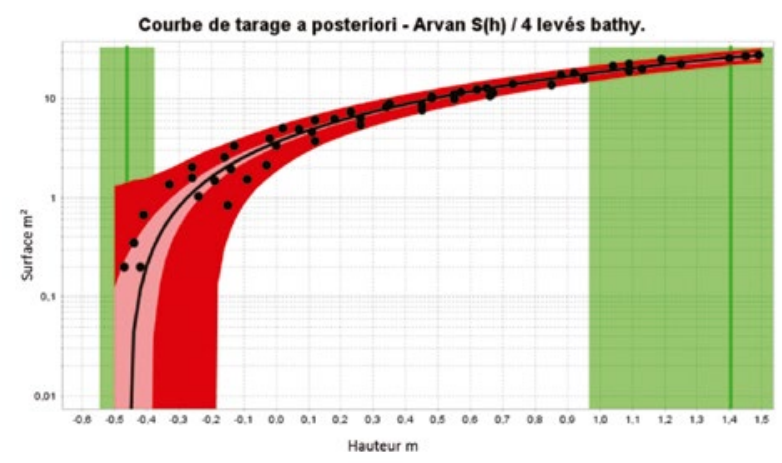

Figure 3b : relation semi-logarithmique hauteur - surface mouillée $S(h)$ pour la station de l'Arvan et fuseau d'incertitude par la méthode BaRatin

La courbe la plus probable et une incertitude associée sont extraites parmi un faisceau de courbes vraisemblables générées par le simulateur bayésien BaRatin. Pour cette station, les évolutions bathymétriques ont davantage d'impact sur les basses valeurs (environ 25\% d'erreur sur S) - que sur les fortes valeurs $(<10 \%)$.

\section{III.3. Implémentation pratique de la relation $V_{m o y}\left(V_{i}\right)$ avec exemples}

Nous proposons de suivre l'approche expérimentale usuelle basée sur l'étude de jaugeages qui permet de prendre en compte l'intégration verticale sur la profondeur et l'intégration latérale sur la section [Jacob, 2014]. On peut aussi vérifier que la vitesse mesurée est représentative de la vitesse débitante sur la section. Le guide d'hydrométrie, Manual on Stream Gauging Volume II [OMM, 2010] présente plusieurs types de relation : affine, polynomiale ou encore linéaire par morceaux. Dans le cas le plus simple i.e. un contrôle hydraulique homogène, la relation $V_{\text {moy }}\left(V_{i}\right)$ est affine. Par exemple, sur la Saône à Lyon, Le Coz et al. [2008] ont utilisé 18 jaugeages ADCP pour construire une relation de très bonne qualité entre la vitesse moyenne et la vitesse témoin d'un H-ACDP, pour une large gamme de vitesses d'écoulement variant de $0,5 \mathrm{~m} / \mathrm{s}$ à plus de $2,5 \mathrm{~m} / \mathrm{s}$ soit un facteur 5 .

Afin d'estimer les incertitudes sur la relation $V_{\text {mov }}\left(V_{i}\right)$, nous avons utilisé sur une période de stabilité la méthode BaRatin sur le cours d'eau torrentiel Arvan à Saint Jean de Maurienne en forçant l'emploi d'une relation linéaire (figure $4 \mathrm{a}$ ). Nous 
avons utilisé d'une part des données $V_{\text {moy }}$ issues de jaugeages et affectées d'une incertitude de 10 à $20 \%$ selon la méthode de jaugeage utilisée et d'autre part des données radar $V_{i}$ affectées d'une incertitude de $10 \%$. La courbe la plus probable et une incertitude associée sont extraites parmi un faisceau de courbes vraisemblables. Le nombre important de jaugeages sur la période étudiée conduit à un niveau d'incertitude quasi constant dans la zone jaugée (de 10 à 15\%). En revanche à bas débit, l'absence de jaugeage se traduit par un fuseau d'incertitude beaucoup plus large $(\sim 50 \%)$.

Sur la Claduègne à Villeneuve de Berg, rivière des monts d'Ardèche, le scénario le plus vraisemblable retient une relation en 3 parties (figure $4 b$ ):

- à l'étiage, la vitesse radar est peu représentative de la vitesse moyenne de l'écoulement et par conséquent le fuseau d'incertitudes est assez large ( $50 \%)$. Une des explications viendrait du fait que chaque mesure radar a été affectée d'une incertitude de $0,10 \mathrm{~m} / \mathrm{s}$, incertitude assez grande proportionnellement à la valeur mesurée. Toutefois cette grande incertitude peut être le reflet de l'instabilité de la relation $V_{m o v}(V)$ à bas débit,

- en régime de base, le fuseau d'incertitude est réduit $(\sim 20 \%)$ notamment parce que les jaugeages sont nombreux et bien répartis dans la gamme,

- en crue, l'inflexion de la courbe traduit la moindre augmentation de la vitesse moyenne par rapport au débit. Ici

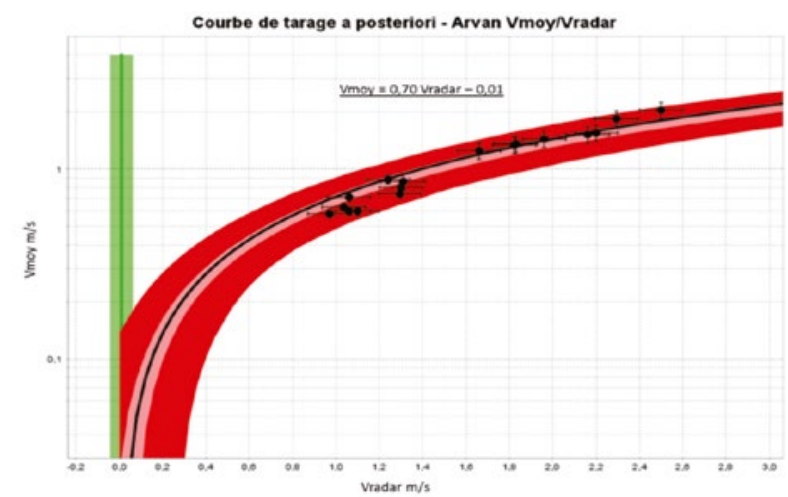

Figure 4a : Relation linéaire $V_{\text {mov }}\left(V_{i}\right)$ semi-logarithmique et fuseau d'incertitude à $95 \%$, pour la station de l'Arvan à $S t$ Jean de Maurienne l'incertitude est légèrement plus importante sans doute par manque de jaugeages. Mais aussi parce que chaque jaugeage a une incertitude propre plus élevée en lien avec la méthode utilisée (jaugeages de surface) et aussi parce qu'ils correspondent à des crues assez rares et des débits très élevés, avec des vitesses radar jusqu'à $5 \mathrm{~m} / \mathrm{s}$.

\section{III.4. Détermination du débit}

Comme pour la méthode courbes de tarage simples $Q(h)$, la chronique de débit est déterminée pour chaque période de stabilité en appliquant l'équation 1 et, selon qu'elle soit unique ou composite, une ou plusieurs relations $V_{\text {mov }}(V)$ établies par analyse de jaugeages. La gamme de débit jaugée détermine la plage d'application de la relation de vitesse témoin.

En pratique, pour la station hydrométrique sur l'Arvan à St Jean de Maurienne, le logiciel libre d'analyses statistiques $\mathrm{R}$ est utilisé pour :

1. interpoler au même pas de temps des chroniques mesurées $h(t)$ et $V_{i}(t)$

2. générer la chronique $S(t)$ par intégration de la bathymétrie

3. appliquer la relation $V_{\text {moy }}\left(V_{i}\right)$

4. calculer le débit $Q(t)$

La figure 5 extraite de la base de données BDOH [Branger et al., 2014] présente un extrait de la chronique $Q(t)$ sur

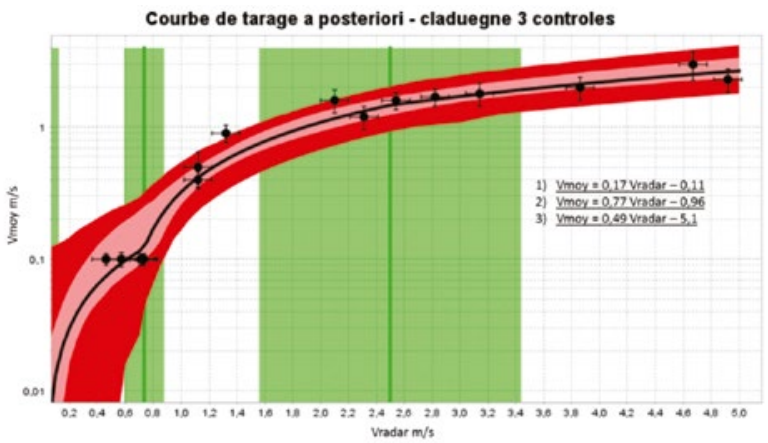

Figure 4b : Relation linéaire $V_{\text {mov }}\left(V_{i}\right)$ semi-logarithmique et fuseau d'incertitude, pour la station de la Claduègne à Villeneuve de Berg

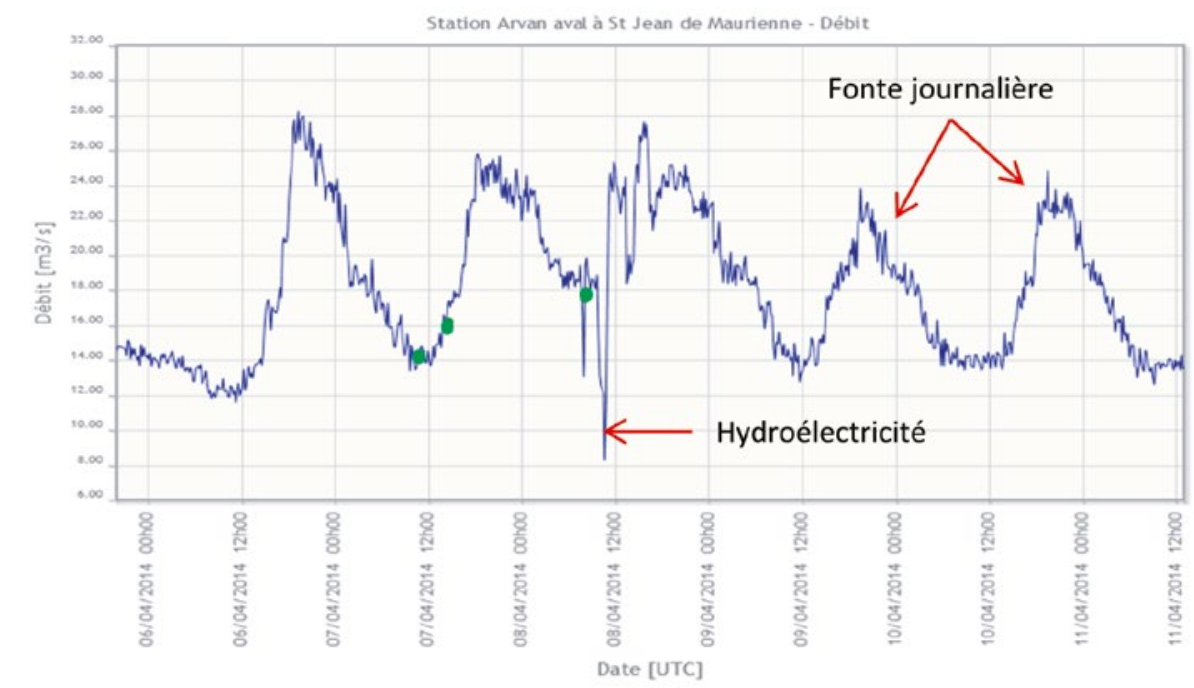

Figure 5 : chronique $Q(t)$ sur l'Arvan à St Jean de Maurienne 
laquelle les jaugeages effectués sur la période, matérialisés par des points verts, sont utilisés comme points de contrôle [Thollet et al. 2016]. Cette représentation permet de s'assurer que la relation utilisée pour le calcul du débit est bien tarée.

Pour le calcul du débit en temps réel, les logiciels des matériels radar récents proposent un calcul de la section mouillée par intégration de la bathymétrie sous la forme d'une table abscisses / profondeurs [Sommer, 2012]. Le calcul du débit est alors effectué directement dans l'appareil, avec prise en compte de la relation $V_{\text {mov }}\left(V_{i}\right)$ préalablement déterminée. Cette solution est à retenir pour une station hydrométrique stable, dont la bathymétrie et la relation $V_{m o v}(V)$ sont bien connues et ne varient pas dans le temps. Dans le cas contraire, suite à un détarage par exemple, le rétro-calcul risque d'être compliqué en dehors de l'appareil.

\section{EXPLOITATION DES MESURES}

\section{IV.1. Détection des détarages}

Comme pour toute station hydrométrique la mesure du débit par radar de surface est liée à la stabilité du contrôle hydraulique. Une variation de bathymétrie peut modifier d'une part la relation hauteur / surface mouillée de la section de mesure (section du radar) et d'autre part, mais plus rarement, la relation $V_{m o y}\left(V_{i}\right)$ liée à la section de mesure et à la section de contrôle en aval (figure $1 \mathrm{~b}$ ). Les variations bathymétriques pour la station sur l'Arvan sont mises en regard des couples $V_{\text {moy }}(V)$ sur les figures $6 \mathrm{a}$ et $6 \mathrm{~b}$. On note que la station détare très fréquemment entre 2011 et 2015 avec une amplitude pouvant atteindre $40 \mathrm{~cm}$ localement. Dans le même temps, c'est seulement après de nombreuses évolutions morphologiques à partir de 2014 que la relation $V_{\text {mov }}(V)$ change : le changement de bathymétrie n'induit pas donc nécessairement sa modification. Dès lors, le recalage d'une station radar semble plus aisé qu'une station $Q(h)$ et principalement lié à la réalisation d'un nouveau levé bathymétrique.

L'utilisation des radars permet de suivre la relation hauteur / vitesse dans le temps. La variation de la relation $h(V)$ est caractéristique d'un détarage et implique la nécessité de nouvelles mesures bathymétriques et la vérification de la stabilité de la relation $V_{\text {moy }}\left(V_{i}\right)$. La réalisation de jaugeages réguliers reste pertinente pour vérifier la cohérence des débits calculés par la méthode de la vitesse témoin, mais pas critique pour la production de chronique $Q(t)$.

Ceci étant, la méthode de la vitesse témoin apparaît plus pratique pour les sites sujets à détarages fréquents que la courbe de tarage hauteur-débit classique, car :

- la relation $V_{m o v}\left(V_{i}\right)$ est moins sensible aux évolutions morphologiques que la relation $Q(h)$;

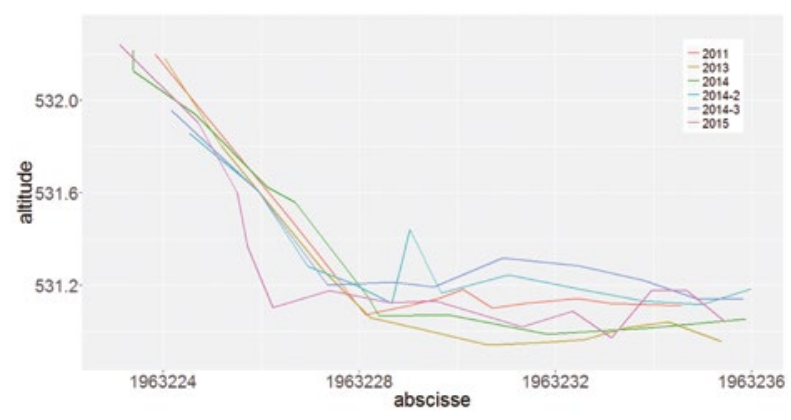

Figure 6a : variation de la bathymétrie de la section de mesure de la station de St Jean de Maurienne
- c'est surtout la relation géométrique $S(h)$ qui est affectée, et elle peut être actualisée avec seulement un levé bathymétrique de la section, sans attendre de disposer d'un jeu de jaugeages sur une gamme étendue de hauteurs.

\section{IV.2. Incertitudes}

Les incertitudes sur le débit par la méthode de la vitesse témoin sont liées au site de mesure et aux conditions de déploiement du matériel. Il est donc très difficile de proposer une méthode standard qui inclue toutes les sources d'incertitudes. Nous nous limiterons ici à lister les sources d'incertitudes de manière non exhaustive :

- Intensité de l'écho du signal rétrodiffusé par la surface de l'écoulement

- Position du radar et représentativité de la vitesse radar par rapport à la vitesse moyenne

- Exposition au vent

- Incertitudes liées aux erreurs aléatoires et systématiques sur les instruments de mesure de hauteur et de vitesse

- Méthode de calcul de la relation $V_{m o y}(V)$ comme le choix du type de relation

- Nombre de jaugeages pour établir cette relation, étendue de la gamme jaugée, incertitude des jaugeages selon la (les) méthode(s) utilisée(s)

- Précision des mesures bathymétriques et méthode de calcul de la surface mouillée

- Stabilité du contrôle hydraulique de la section de mesure et fréquence des bathymétries de contrôle...

Lors de l'étude hydraulique de la station hydrométrique sur l'Arvan, [Jacob, 2014] a proposé un calcul basé sur la propagation des incertitudes selon le GUM [JCGM 2008]. L'étude a montré que l'incertitude sur le débit reste modérée, typiquement $10 \%$, pour les périodes de stabilité du contrôle hydraulique et où les données de bathymétrie sont bien connues. L'incertitude est néanmoins plus forte à très bas et très haut débit en lien avec le manque de jaugeages. La station étudiée étant à lit particulièrement instable et les données de bathymétrie difficiles à acquérir hors étiage, l'incertitude moyenne sur le débit est de l'ordre de 30\% sur l'ensemble des données. L'étude a aussi permis de comparer le débit calculé avec la méthode classique de détermination du débit $Q(h)$. Elle a montré une moindre sensibilité aux détarages ainsi qu'une plus grande facilité à établir une nouvelle relation puisque seule une nouvelle bathymétrie est nécessaire pour réinitialiser le calcul du débit, contrairement à la méthode $Q(h)$ où de nombreux jaugeages sont demandés.

La méthode bayésienne BaRatin nous a permis de caler les relations $V_{\text {moy }}\left(V_{i}\right)$ et $S(h)$ pour les stations sur l'Arvan et la Claduègne et de proposer une première évaluation des incertitudes associées. La généralisation de cette approche

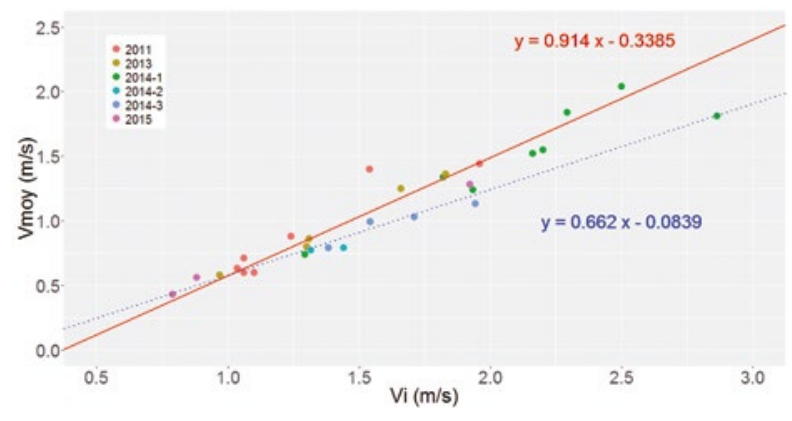

Figure 6b : variation de la relation $V_{\text {moy }}\left(V_{i}\right)$ avec poistionnement des jaugeages liés à leurs bathymétries 
à la méthode de la vitesse témoin peut être envisagée pour l'avenir : il faudra alors gérer deux relations calées avec des mesures indépendantes (jaugeages et bathymétries).

\section{CONCLUSIONS}

La détermination du débit par la méthode de la vitesse témoin semble particulièrement appropriée aux cours d'eau torrentiels, souvent difficiles à jauger et où les écoulements turbulents sont favorables aux mesures radar. Des données de calage sont nécessaires au calcul de débit : données bathymétriques pour la relation $S(h)$ et jaugeages pour la détermination de la relation $V_{\text {mov }}(V)$. Par rapport aux méthodes traditionnelles, elle présente l'avantage de mieux documenter les hydrogrammes en crue, d'être plus facile et rapide à recaler après un détarage.

Le calcul du débit en temps réel est possible et le post-traitement des données permet de détecter les non stationnarités (détarages) de la station en suivant l'évolution de la relation $V_{i}(h)$.

L'utilisation plus large de la méthode et le développement des outils de mesure permettra à l'avenir de mieux évaluer l'incertitude liée au calcul du débit. Une généralisation de la méthode bayésienne BaRatin pour traiter ce type spécifique de courbes de tarage pour l'aire mouillée et la vitesse moyenne est envisagée pour les prochaines années.

\section{REMERCIEMENTS}

Ce travail a été réalisé en s'appuyant sur des données des stations de mesure appartenant aux réseaux ZABR, OHMCV et Hymex.

Les auteurs remercient également les équipes Irstea de développement pour les outils BaRatin (Benjamin Renard), Phamhyr (Jean-Baptiste Faure) et BDOH (Nicolas Raidelet, Luc Jamet et Guillaume Perréal).

\section{RÉFÉRENCES}

Birgand F., Benoist J.C., Novince E.; Gilliet N., Saint-Cast P., LE SAOS E. (2005) - Mesure des débits à l'aide de débitmètres ultrasoniques Doppler. Cas des petits cours d'eau ruraux. Ingénieries - E A T, 41, 23-38.

Branger F., Thollet F., Crochemore M., Poisbeau M., Raidelet N., Farissier P., Lagouy M., Dramais G., Le Coz J., Guerin A., Tallec G., Peschard J., Mathys N., Klotz S., Tolsa M. (2014) - Le projet Base de Données pour les Observatoires en Hydrologie : un outil pour la bancarisation, la gestion et la mise à disposition des données issues des observatoires hydrologiques de long terme à Irstea. Houille Blanche-Revue Internationale de l'Eau, 1, 33-38, doi: 10.1051//hb/2014005.

Costa J.E., Chang R.T., Haeni F.P., Melcher. N., Teague C., AND BARRISCK D. (2006) - Use of radars to monitor stream discharge by noncontact methods. Water Resources Research, 42, W07422.

Dramais G., Le Coz J., Camenen B., Hauet A. Duby P. (2011) Utilisation des mesures sans contact pour quantifier le débit des rivières torrentielles. Colloque SHF : "L'eau en montagne: mieux observer pour mieux prévoir », Lyon, 16-17 mars 2011, $11 \mathrm{p}$.

Hauet A., Le Coz J.; Dramais G.; Carre C.; Legras D.; Pierrefeu G.; Godayer C. (2009) - Méthodes innovantes pour la mesure des débits fluviaux en continu : profileur Doppler fixe horizontal (H-aDcp) et analyse d'images (LSPIV). Houille Blanche-Revue Internationale de l'eau, 3, 123-131, doi 10.1051/lhb/2009036.

JACOB E. (2014) - Débits et incertitudes issus d'enregistrements de niveau et de vitesse par radar sur des cours d'eau de montagne à lit instable. Mémoire de stage, Irstea, $60 \mathrm{p}$.

JCGM (2008) - Evaluation des données de mesure. Guide pour l'expression de l'incertitude de mesure, 132p.

Le Coz J., Renard B., Bonnifait L., (2014) - Combining hydraulic knowledge and uncertain gaugings in the estimation of hydrometric rating curves: A Bayesian approach. 509(13), 573-587.

Le Coz J., Pierrefeu G., Paquier A. (2008) - Evaluation of river discharges monitored by a fixed side-looking Doppler profiler. Water Resources Research, 44, W00D 09.

Le Coz J., Chaleon C., Bonnifait L., Le Boursicaud L., Renard B., Branger F., Diribarne J., Valente M. (2013) - Analyse bayésienne des courbes de tarage et de leurs incertitudes : la méthode BaRatin. Houille Blanche-Revue Internationale de l'Eau, 6, 31-41, doi 10.1051/lhb/2013048.

LeVesque V.A., OBerg K.A. (2012) - Computing Discharge Using the index velocity method, USGS, Virginia, 46 pages.

Nord G., Gallart F., Gratiot N., Soler M., Reid I., Vachtman D., Latron J., Martín Vide J.P. And LaRonne J.B. (2014) Applicability of acoustic Doppler devices for flow velocity measurements and discharge estimation in flows with sediment transport. Journal of Hydrology, 509, 504-518, doi 10.1016/j. jhydrol.2013.11.020.

Nord G., Boudevillain B., Berne A., Branger F., Braud I., Dramais G., Gérard S., Le Coz J., Legoût C., Molinié G., Van Baelen J., Vandervaere, Jp, Andrieu J., Aubert C., Calianno M., Delrieu G., Grazioli J., Hachani S., Horner I., Huza J., Le Boursicaud R., Raupach T., Teuling A., Uber M., Vincendon B.; WiJbrans A. (2016) - A high space-time resolution dataset linking meteorological forcing and hydro-sedimentary response in a mesoscale Mediterranean catchment (Auzon) of the Ardèche region, France. Earth Syst. Sci. Data Discuss, doi 10.5194/essd-2016-32.

Thollet, F, Camenen B.; Le Coz J.; Dramais G. (2016) - Extrait de la base de données BDOH, doi 10.17180/OBS.ARC-ISERE.

Welber M., Le Coz J., Laronne J.B., Zolezzi G., Zamler D., Dramais G., Hauet A., Salvaro M. (2016) - Field assessment of non-contact stream gauging using portable surface velocity radars (SVR). Water Resources Research, 52, 1108-1126 doi 10.1002/2015WR017906.

World Meteorological Organization (2010) - Manual on Stream Gauging Computation of discharge, volume II. 